\title{
Impact of a single distance phase retrieval algorithm on spatial resolution in X-ray inline phase sensitive imaging
}

\author{
Muhammad U. Ghani ${ }^{\mathrm{a}, *}$, Bradley Gregory ${ }^{\mathrm{a}}$, Farid Omoumi ${ }^{\mathrm{a}}$, Bin Zheng ${ }^{\mathrm{a}}$, Aimin Yan ${ }^{\mathrm{b}}$, \\ Xizeng $\mathrm{Wu}^{\mathrm{b}}$ and Hong Liu ${ }^{\mathrm{c}}$ \\ ${ }^{a}$ Advanced Medical Imaging Center and School of Electrical and Computer Engineering, University of \\ Oklahoma, Norman, OK 73019, USA \\ E-mails: usman87@ou.edu,bradley.gregory@ou.edu,farid.h.omoumi@ou.edu,Bin.Zheng-1@ou.edu \\ ${ }^{\mathrm{b}}$ Department of Radiology, University of Alabama at Birmingham, Birmingham, AL, 35249, USA \\ E-mails:ayan@uabmc.edu,xwu@uabmc.edu \\ ${ }^{\mathrm{c}}$ Advanced Medical Imaging Center and School of Electrical and Computer Engineering, University of \\ Oklahoma, Norman, OK 73019, USA \\ E-mail:liu@ou.edu
}

\begin{abstract}
A single-projection based phase retrieval method based on the phase attenuation duality principle (PAD) was used to compare the spatial resolution of the acquired phase sensitive and PAD processed phase retrieved images. An inline phase sensitive prototype was used to acquire the phase sensitive images. The prototype incorporates a micro-focus x-ray source and a flat panel detector with a $50 \mu \mathrm{m}$ pixel pitch. A phantom composed of a $2 \mathrm{~cm}$ thick $50-50$ adipose-glandular mimicking slab sandwiched with a $0.82 \mathrm{~cm}$ thick slanted PMMA sharp edge was used. Phase sensitive image of the phantom was acquired at $120 \mathrm{kV}, 3.35 \mathrm{mAs}$ with a $16 \mu \mathrm{m}$ tube focal spot size under a geometric magnification (M) of 2.5. The PAD based method was applied to the acquired phase sensitive image for the retrieval of phase values. With necessary data processing, modulation transfer function (MTF) curves were determined for the estimation and comparison of the spatial resolution. The PAD processed phase retrieved values of the phantom were in good agreement with the theoretically calculated values. Phase sensitive images showed higher spatial resolution at all spatial frequencies compared to the phase retrieved images. It was noted that the highfrequency signal components in the retrieved image were suppressed that resulted in lower MTF values. When compared to the phase sensitive image, the cutoff resolution (10\% MTF) for phase retrieved image dropped $32 \%$ from $15.6 \mathrm{lp} / \mathrm{mm}(32 \mu \mathrm{m})$ to $10.6 \mathrm{lp} / \mathrm{mm}(47 \mu \mathrm{m})$. The resolution offered by this phase sensitive prototype is radiographically enough to detect breast cancer.

Keywords: Phase retrieval, high-energy phase contrast imaging, spatial resolution
\end{abstract}

\section{Key Abbreviations and Symbols}

(PAD) Phase Attenuation Duality

(FPP) Fresnel propagator phase

( $\beta$ Linear attenuation coefficients

( $\delta) \quad$ Refractive index decrement

\footnotetext{
*Corresponding author: Muhammad Usman Ghani, Postdoctoral Research Fellow, Electrical and Computer Engineering, The University of Oklahoma. E-mail: usman87@ou.edu.
} 
$\left(\rho_{e, p}\right) \quad$ Projected electron density

(MTF) Modulation Transfer Function

\section{Introduction}

In-line phase sensitive imaging technique (also known as propagation-based phase sensitive imaging) has demonstrated its value as compared to the attenuation-based imaging [1-8]. Experimentally, this technique is the simplest to because its setup does not involve any optical element introduced between the sample and detector [9-11]. However, data processing required for extracting an object's phase map from the measured intensity is very complicated for this technique. Theoretically, the x-ray intensity arriving at the detector plane $\mathrm{I}\left(\overrightarrow{r_{D}}\right)$ is given by the general X-ray transport equation [12-14]

$$
\begin{aligned}
I\left(\overrightarrow{r_{D}}\right)= & \frac{I_{\text {in }}}{M^{2}}\left\{\cos \left(\frac{\lambda R_{2}}{4 \pi M} \nabla^{2}\right)\left[A^{2}(\vec{r})+\frac{\lambda R_{2}}{2 \pi M}\left(\nabla \cdot\left(\phi(\vec{r}) \nabla A^{2}(\vec{r})\right)\right)\right]\right. \\
& \left.-2 \sin \left(\frac{\lambda R_{2}}{4 \pi M} \nabla^{2}\right)\left[A^{2}(\vec{r}) \phi(\vec{r})\right]\right\}
\end{aligned}
$$

where $\lambda$ represents the x-ray wavelength, $I_{\text {in }}$, the entrance intensity at the object plane, $R_{2}$, the objectdetector distance, $\mathrm{M}$, the geometric magnification factor while $A^{2}(\vec{r})$ and $\phi(\vec{r})$ are the attenuation map and phase shift map of the object. Furthermore, $\cos \left(\frac{\lambda R_{2}}{4 \pi M} \nabla^{2}\right)$ and $\sin \left(\frac{\lambda R_{2}}{4 \pi M} \nabla^{2}\right)$ are the 2D pseudodifferential operators in which $\nabla^{2}$ is the Laplacian operator. The general x-ray transport equation is the basis for developing phase retrieval methods. The equation can be simplified for many applications where the maximal Fresnel propagator phase $\left(F P P_{M}=\pi \lambda R_{2} / 4 M p^{2}\right)$ is much less than $1[13,14]$, where $\mathrm{p}$ represents the pixel size of the detector. If an imaging setup employs a detector pixel size on the order of tens of micrometers and a sample-detector distances of a few meters or less, then the corresponding maximal Fresnel propagator phase $F P P_{M} \ll 1$. Under these conditions, the 2D pseudodifferential operators become constants $[12,13]$ and Eq. (1) reduces to

$$
I\left(\overrightarrow{r_{D}}\right)=\frac{I_{\text {in }}}{M^{2}}\left\{A^{2}(\vec{r})-\frac{\lambda R_{2}}{2 \pi M}\left(\nabla \cdot\left(A^{2}(\vec{r}) \nabla \phi(\vec{r})\right)\right)\right\}
$$

Both the attenuation and phase maps are differentially encoded with each other in the projection intensity $I\left(\overrightarrow{r_{D}}\right)$. Hence, for the task of retrieving the phase map of an object, one should decode the phase information from the projection views by utilizing the above equations. In general, phase retrieval requires the acquisition of at least two projections with varying object-detector distances per projection [9,15-17]. This adds complexity to the experiments and increases the radiation dose delivered to biomedical samples. There is continuing interest and research in developing the so-called single-distance or single-projection acquisition techniques for retrieving the phase maps in diagnostic imaging [18-23]. However, if a single-projection acquisition technique is used, stringent validity conditions are required to decode the contributions of attenuation and phase.

One of the methods of single-projection-based retrieval works correctly for a single material sample if the linear attenuation coefficients $(\beta)$ and refractive index decrement $(\delta)$ of that material are known in advance [23]. In such special cases, the phase map of a single-material sample can be retrieved from just a single-phase sensitive projection of the sample. While this phase retrieval method is robust, the 
stringent requirement on the material homogeneity and prior knowledge of material's $\delta$ and $\beta$ limits its applicability. Another single-projection based retrieval method that utilizes the phase-attenuation duality (PAD) principle has been developed [24-26] and has been applied in various medical imaging studies [27-35]. This method is applicable when samples that are composed of light chemical elements with atomic number $(Z)<10$ are imaged with high energy $x$-rays $(60-500 \mathrm{keV})$. With this method, one can retrieve the sample's phase map using just a single-phase sensitive projection of the sample, without requiring any prior information of the sample's $\delta$ and $\beta$ at all. Another advantage of this method is its applicability with the multi-material samples. When low $\mathrm{Z}$ materials are imaged with high-energy $\mathrm{x}$-rays, the $\mathrm{x}$-ray-matter interactions are dominated by the Compton scattering. Hence, both the tissue attenuation $\left(A_{\mathrm{KN}}^{2}\right)$ and phase shifts $(\phi)$ are all uniquely related to the projected electron density $\left(\rho_{e, p}\right)$ distributions as

$$
\begin{aligned}
& A_{\mathrm{KN}}^{2}(\vec{r})=\exp \left(-\sigma_{\mathrm{KN}} \rho_{e, p}(\vec{r})\right) \\
& \left.\phi(\vec{r})=-\lambda r_{e} \int \rho_{e}(\vec{r}, z) d r=-\lambda r_{e} \rho_{e, p} \overrightarrow{(r}\right)
\end{aligned}
$$

Under such conditions, the phase-shift map can be retrieved from just a single-phase sensitive image as

$$
\begin{aligned}
& A_{\mathrm{KN}}^{2}(\vec{r})=\left\{\left[1-\left(\frac{\lambda^{2} R_{2} r_{e}}{2 \pi M \sigma_{\mathrm{KN}}} \nabla^{2}\right)\right]^{-1}\left(\frac{M^{2}}{I_{\mathrm{in}}} I\left(\overrightarrow{r_{d}}\right)\right)\right\} \\
& \phi(\vec{r})=\frac{\lambda r_{e}}{\sigma_{\mathrm{KN}}} \cdot \ln A_{\mathrm{KN}}^{2}(\vec{r})
\end{aligned}
$$

where, $r_{e}=2.818 \times 10^{-15} \mathrm{~m}$ is the classical electron radius and $\sigma_{\mathrm{KN}}$ is the total cross-section for x-ray photon Compton scattering from a single free electron derived from the Klein Nishina formula as [25]

$$
\sigma_{\mathrm{KN}}=2 \pi r_{e}^{2}\left\{\frac{1+\eta}{\eta^{2}}\left[\frac{2(1+\eta)}{1+2 \eta}-\frac{1}{\eta} \log (1+2 \eta)\right]+\frac{1}{2 \eta} \log (1+2 \eta)-\frac{(1+3 \eta)}{(1+2 \eta)^{2}}\right\}
$$

In Eq. (7), $\eta=E_{\text {photon }} / m_{e} c^{2}, E_{\text {photon }}$ is the photon energy of the primary x-ray beam, $m_{e} c^{2}$ is the resting electron energy and $r_{e}$ is the classical electron radius.

With the application of any image processing method on medical images, the impact of spatial resolution must be investigated. To the best of our knowledge, no comprehensive investigation of the impact of PAD based method on the spatial resolution has been performed. In this study, a phantom's phase values retrieved experimentally by the PAD-based method are compared with the theoretically calculated phase values. Furthermore, the impact of this retrieval method on spatial resolution is studied by measuring the modulation transfer function (MTF). The measurement of MTF has been widely accepted for characterizing and quantifying the imaging performance of various imaging modalities. The calculation of MTF can be determined by normalizing the absolute value of the Fourier transform of the point spread function (PSF) or line spread function (LSF). By utilizing a sharp edge phantom, the edge spread function (ESF) can be acquired and then differentiated to obtain the LSF. 


\section{Materials and methods}

\subsection{Phase-sensitive imaging prototype}

A benchtop inline phase sensitive $\mathrm{x}$-ray imaging prototype was used for the acquisition of the phantom images. Details on the characterization of this prototype can be found elsewhere [29,30]. In summary, the prototype incorporates a microfocus $\mathrm{x}$-ray source with a tungsten $(\mathrm{W})$ target and a CMOS flat panel detector with an active area of $120 \mathrm{~mm} \times 120 \mathrm{~mm}$ and $50 \mu \mathrm{m}$ pixel pitch. The source to object distance was $68 \mathrm{~cm}$ while the source to image detector distance was $170 \mathrm{~cm}$ which corresponded to a magnification $(M)$ factor of 2.5 . The phase sensitive images of the phantom were acquired at $120 \mathrm{kV}, 67 \mu \mathrm{A}$, $3.3 \mathrm{mAs}$ with a focal spot (F) size of $16 \mu \mathrm{m}$. A $2.5 \mathrm{~mm}$ thick aluminum (Al) filter was utilized to harden the beam and block the low energy photons. These specific geometric and acquisition parameters are the optimized settings for this prototype operating with high energy x-rays for the acquisition of soft tissue and its equivalent materials [36]. In utilizing the polychromatic microfocus $\mathrm{X}$-ray tube, it was necessary to approximate the values of the average wavelength $(\lambda)$ and the Klein-Nishina total cross-section $\left(\sigma_{\mathrm{KN}}\right)$ utilized in Eqn (5) corresponding to a single wavelength monochromatic x-ray beam. The approximate average polychromatic $\mathrm{x}$-ray output of a tungsten target tube operating at $120 \mathrm{kVp}$ corresponds to a $60 \mathrm{keV}$ monochromatic $\mathrm{x}$-ray beam which gives $\lambda=2.066 \times 10^{-11} \mathrm{~m}$ and $\sigma_{\mathrm{KN}}=5.456 \times 10^{-29} \mathrm{~m}^{2}$. The $F_{P P}$ value corresponding to this imaging setup was 0.00264 , which is much less than 1 and hence the approximation of Eqn (2) are fully valid.

\subsection{Phantom}

A phantom composed of a $2 \mathrm{~cm}$ thick slab mimicking a 50\% glandular-50\% adipose density sandwiched with a $0.82 \mathrm{~cm}$ thick polymethyl methacrylate (PMMA) was used for imaging. Both the 50-50 slab and PMMA were manufactured by Gammex (Middleton, WI, USA). The 50-50 slab is composed of the epoxy resin $\left(\mathrm{C}_{21} \mathrm{H}_{25} \mathrm{CIO}_{5}\right)$ that simulates the attenuation properties of breast tissue to x-rays. The 50-50 slab and PMMA $\left(\mathrm{C}_{5} \mathrm{O}_{2} \mathrm{H}_{8}\right)_{\mathrm{n}}$ are composed of chemical elements with $\mathrm{Z}<10$, thus, the requirement of applying the phase-attenuation duality is readily met. The combination of the 50-50 slab and PMMA makes the phantom multi-material, and the efficacy of this phase retrieval method can be fully realized. Laser cutting technique was used for the fabrication of a sharp edge to ensure reliable measurements of the spatial resolution. The schematics of the phase sensitive prototype along with the image of the phantom are shown in Fig. 1.

\subsection{MTF calculations}

The PMMA edge was aligned to the source focal spot size and was slightly tilted $\left(3^{\circ} \sim 5^{\circ}\right)$ for the generation of oversampled ESF that had a data interval smaller than the size of the pixel pitch [37,38]. Using the Hough transformation [39-41], the edge angle was estimated to be $3.2^{\circ}$ on both phase sensitive and phase retrieved images. A fixed window (W) of 4 elements was convolved with the raw ESF to get the smoothed ESF as

$$
E S F_{S}(X)=\sum_{m=-\infty}^{\infty} W(X-m) \cdot E S F_{\mathrm{Raw}}(X)
$$




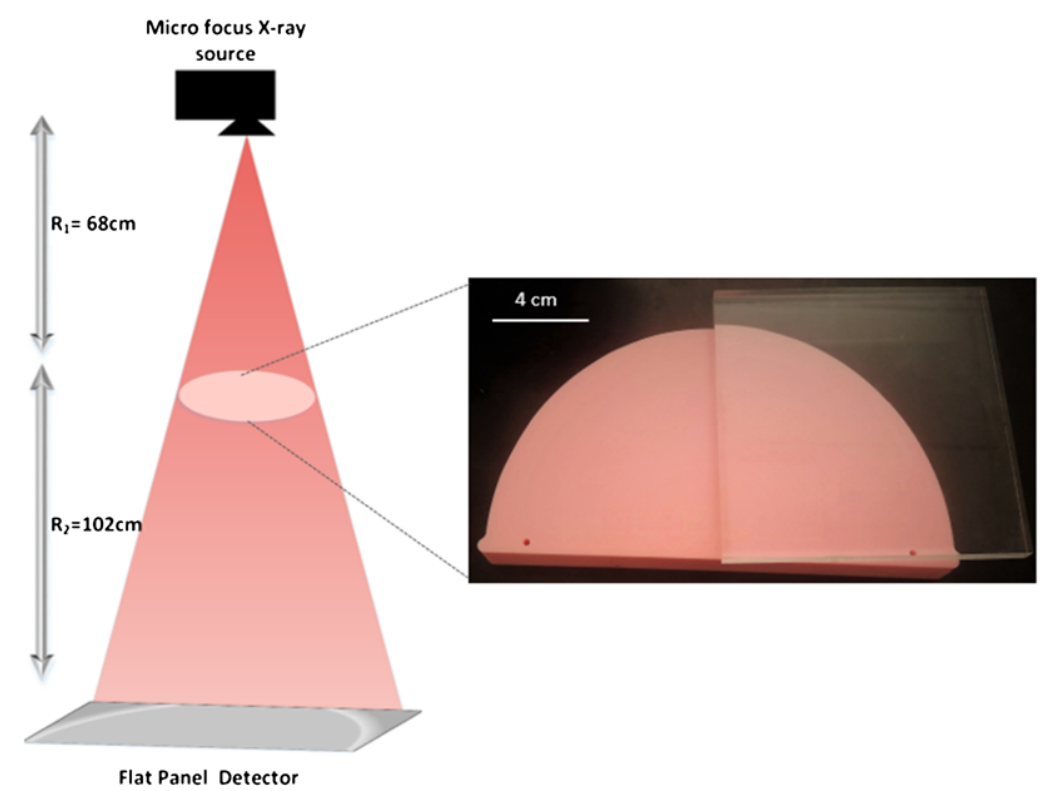

Fig. 1. Schematics of the inline phase sensitive x-ray imaging prototype illustrating a micro-focus x-ray source, a flat panel detector and a $2.82 \mathrm{~cm}$ thick phantom placed in a magnification geometry.

Then, the line spread function, $\operatorname{LSF}(X)$, was computed by differentiating the $\operatorname{ESF}_{S}(X)$ function. A Gaussian function, $F_{G}(X)$, was used to fit the $\operatorname{LSF}(\mathrm{X})$ that made the overall MTF curve better behaved by forcing a smoothing transition of the LSFs to zeros.

$$
F_{G}(X)=\sum_{i=1}^{i=G} a_{i} e^{-\left(\frac{X-b_{i}}{c_{i}}\right)^{2}}
$$

where $a_{i}, b_{i}$ and $c_{i}$ are all constants and $G$ defines the order of the Gaussian function. A 7 th order Gaussian function was used to fit the $\operatorname{LSF}(\mathrm{X})$ obtained from the phase sensitive image and a 3rd order Gaussian function was used to fit the LSF obtained from the phase retrieved image. Finally, the modulation transfer function $(M T F)$ curves were obtained from the fast Fourier transform $(F F T)$ of the LSF, and normalization to unity at zero spatial frequency $(f)$ as [39-41]

$$
\begin{aligned}
& \operatorname{MTF}(f)=\operatorname{FFT}\{\operatorname{LSF}(X)\} \\
& |\operatorname{MTF}(f)|=\operatorname{MTF}(f) / \operatorname{MTF}(0)
\end{aligned}
$$

\section{Results}

The phase sensitive image of the 50-50 slab-PMMA edge phantom acquired by the prototype and the retrieved image $\left(A_{\mathrm{KN}}^{2}\right)$ processed by PAD method are shown in Fig. 2(a) and (b). As shown in Fig. 2(c), the phase map of the phantom was retrieved using the using Eqn. (2) with $A_{\mathrm{KN}}^{2}$ image as it's input.

Note that with the PAD method, the phase retrieved map reported an average value of -389 radians along the 50-50 slab and -593 radians along the slab-PMMA edge. Thus, the average phase value for 


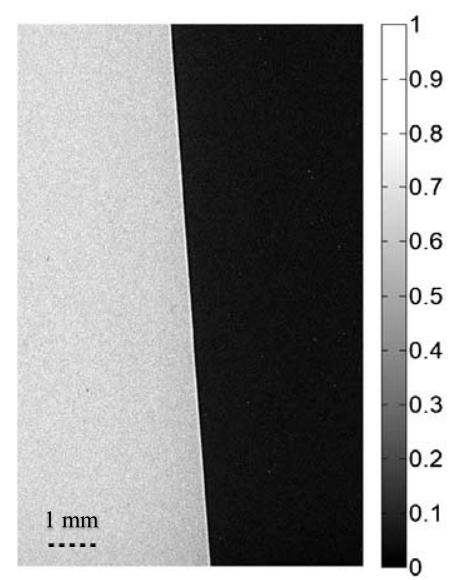

(a)

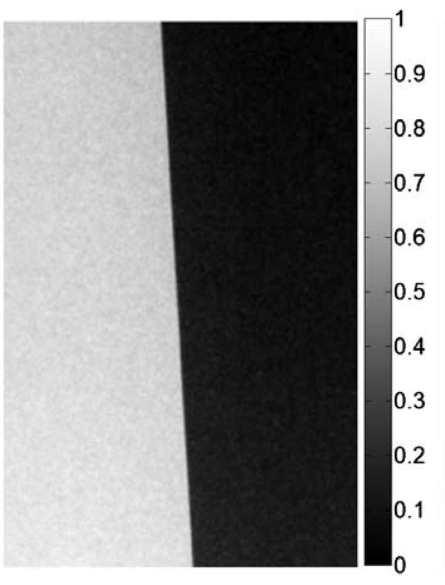

(b)

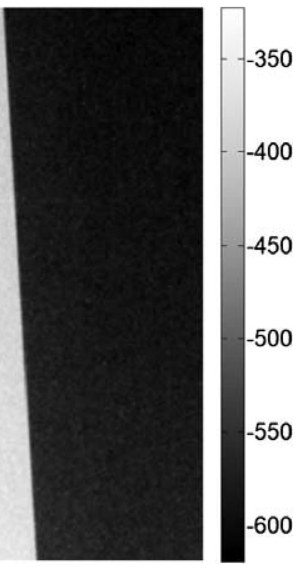

(c)

Fig. 2. (a) phase sensitive image of the 50-50 slab-PMMA edge phantom acquired with the prototype (b) retrieved image $\left(A_{\mathrm{KN}}^{2}\right)$ processed with the PAD based method (c) phase retrieved map of the phantom with the color bar highlighting the retrieved phase values. For 2(a) and 2(b), the color bars highlight the normalized intensities in single-precision floating point numbers.

Table 1

Comparison of theoretical and retrieved phase values for the phantom

\begin{tabular}{cccc}
\hline Material type values (radians) & Theoretical phase values (radians) & Phase retrieved & $\%$ Error \\
\hline 2 cm 50-50 slab & -379 & -389 & 2.63 \\
2.82 cm Combo & -564 & -593 & 5.1 \\
0.82 cm PMMA & -185 & -204 & 10.2 \\
\hline
\end{tabular}

the PMMA edge after their difference is about -204 radians. Note that the $\mathrm{x}$-ray phase shifts should be of negative values, as x-ray refractive indices of tissues and materials are complex, and their real parts are less than one.

Theoretically, the amount of phase shift along a ray can be calculated as $\phi(\vec{r})=-\lambda r_{e} \rho_{e} T_{e}(\vec{r})$, assuming all rays have normal incidence, where $T_{e} \overrightarrow{(r)}$ denotes the projected phantom thickness along the ray path. For a $2 \mathrm{~cm}$ thick 50-50 slab having an electron density $\left(\rho_{e}\right)$ of $3.261 \times 10^{23} / \mathrm{cm}^{3}$, we found that the approximate projected phase shift value along the slab is about -379 radians. Similarly, for the $0.82 \mathrm{~cm}$ thick PMMA edge having an electron density $\left(\rho_{e}\right)$ of $3.874 \times 10^{23} / \mathrm{cm}^{3}$, the phase shift along the edge is about -185 radians and the approximate phase shift along the $2.82 \mathrm{~cm}$ thick slabedge phantom is about -564 radians. Thus, the PAD based method reported the phase shift values that are reasonably close to the thickness-based phase estimates. The comparison of theoretically calculated phase and experimentally retrieved phase values are given in Table 1.

On the phase sensitive image, the overshooting at the boundaries are clearly seen. As the distorted wave-front propagates sufficiently far, the small differences in phase shifts of the wavefront cause interference patterns which causes edge enhancements (dark-bright fringes) at boundaries between components of varying phase shift coefficients. These interference patterns are not visible after the application of the phase retrieval method on the phase sensitive image. The oversampled ESFs evaluated across the sharp PMMA-air edge for both the phase sensitive and PAD processed images are given in Fig. 3. The application of Eq. (6) to raw ESF generates smooth ESF curves with a high coefficient of determination 


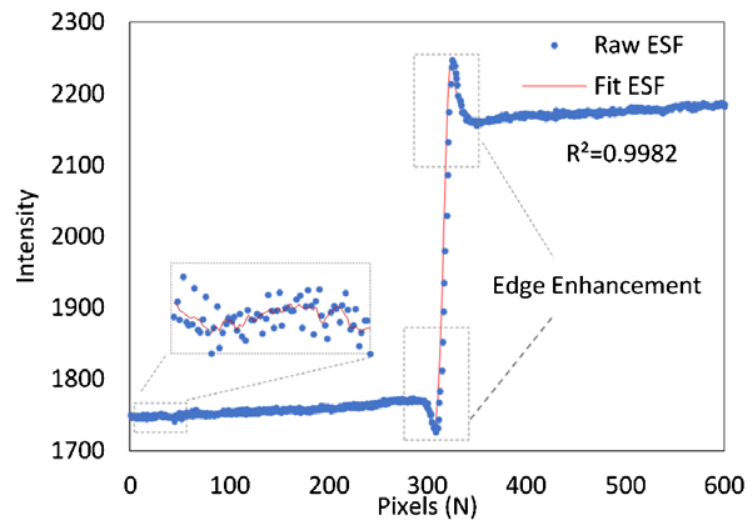

(a)

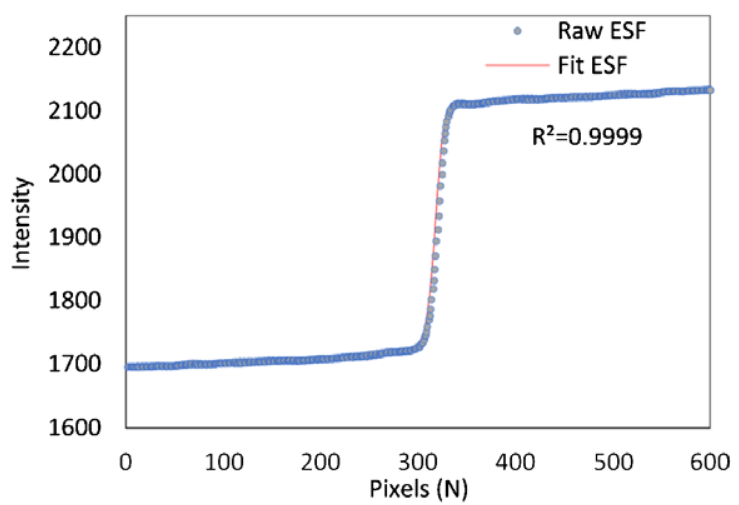

(b)

Fig. 3. Oversampled edge spread functions (ESFs) obtained from the (a) phase sensitive image (b) phase retrieved image. The results of applying the window function to the raw ESF are zoomed in 3(a).

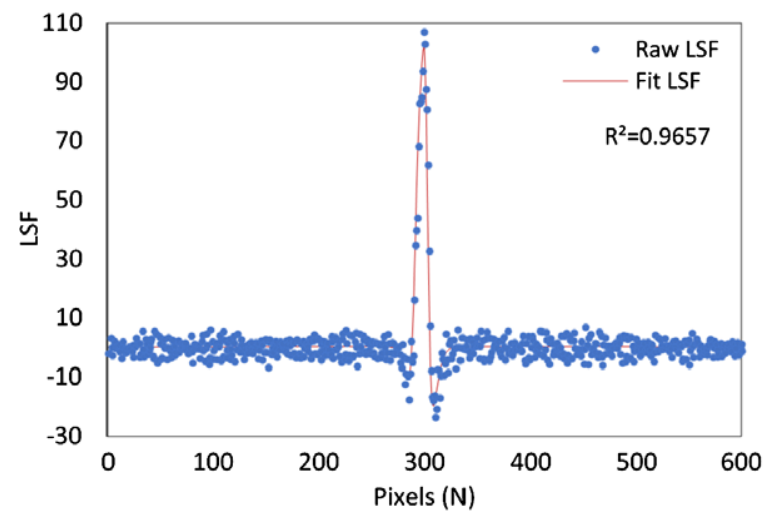

(a)

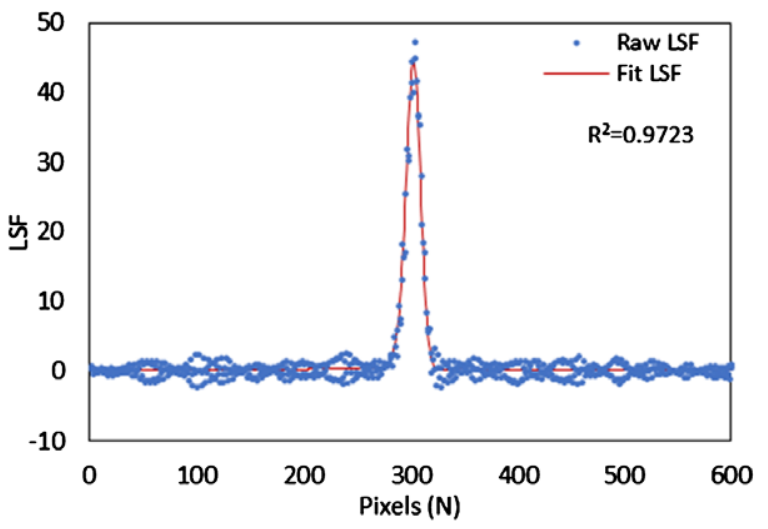

(b)

Fig. 4. Line spread functions (LSFs) obtained for the (a) phase sensitive image (b) phase retrieved image.

$\left(R^{2}\right)$ values while preserving the edge truth. Note that the intensities of both images are converted back to 12-bit grayscale values. The edge enhancement effects are highlighted for the phase sensitive image.

The differentiation of ESF curves produces LSF curves as shown in Fig. 4. The raw LSF curves were fitted using the Gaussian function given by Eqn. (7), generating the fitted LSF curves with high $R^{2}$ values that indicates a good fitting. The edge enhancement as highlighted in the ESF curves for the phase sensitive image causes negative side lobes (dips) in the corresponding LSF curves. The full width at half maximum (FWHM) values on LSF curves of phase sensitive and phase retrieved images were determined to be $209 \mu \mathrm{m}$ and $318 \mu \mathrm{m}$.

The MTF curves for phase sensitive and phase retrieved images are given in Fig. 5. On the phase sensitive image, the interference patterns occurring at the boundaries between components of varying phase shift coefficients results in the amplification of signals in the mid-frequencies. The recorded resolutions for the two images at 50\% MTF are $11.5 \mathrm{lp} / \mathrm{mm}$ and $5.3 \mathrm{lp} / \mathrm{mm}$; while $15.6 \mathrm{lp} / \mathrm{mm}$ and $10.6 \mathrm{lp} / \mathrm{mm}$ are reported at $10 \% \mathrm{MTF}$ for the phase sensitive and phase retrieval imaging. 


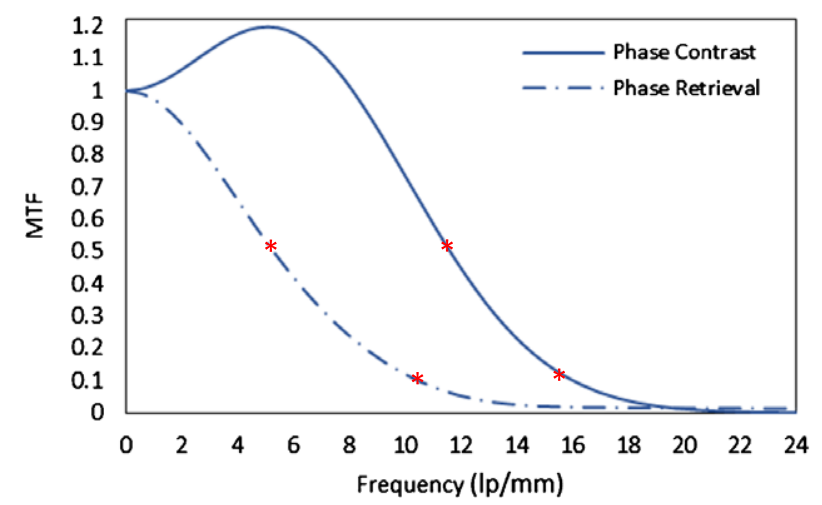

Fig. 5. Modulation transfer function (MTF) curves for the phase sensitive and phase retrieved images. The asterisk $(*)$ signs show the frequencies corresponding to $50 \%$ and $10 \%$ MTF values.

\section{Discussion}

Phase-contrast imaging has shown its value in the preclinical imaging studies, and there has been a rapid acceleration over the past few years towards its clinical implementation [7,9,42-45]. Experimentally, the in-line phase sensitive imaging is the most straightforward technique to implement. A necessary sample to detector distance is required to let the distorted wave propagate and diffract. This technique also works with polychromatic x-ray sources. One of the important goals for phase sensitive imaging is to get quantitative information such as tissue's electron density and phase retrieval methods can yield that information. Depending on the sample's composition, phase retrieval methods can be applied to a single-phase sensitive image. One such method is based on phase-attenuation duality (PAD) principle.

Without the prior information of $\delta$ and $\beta$ of the multi-material phantom, PAD based method retrieved the phase numbers of the phantom which were within $2-10 \%$ of the theoretically calculated phase values. It was noted that with the PAD-based method, high-frequency signal components in the retrieved image were suppressed that resulted in lower MTF values. Mathematically, the Laplacian operator in Eqn (4) makes the denominator very large, thus, excessively suppressing high-frequency components in the resultant image. For example, the cutoff resolution (10\% MTF) suggest that the smallest detectable details on phase retrieved and phase sensitive images were $47 \mu \mathrm{m}$ and $31 \mu \mathrm{m}$. Yet, the retrieved image's noise power spectrum (NPS) suggests that there is also suppression of high-frequency noise component. The comparison of NPS is beyond the scope of this topic and will be presented in a future study. It has been shown in the previous studies that the PAD-based method yielded images with an improvement of at least three times higher contrast to noise ratios (CNRs) than the phase sensitive images of biological samples [29-33]. In this study, the CNR values for the phantom were 24 and 66 for the phase sensitive and phase retrieved images.

The polychromatic beam has a contribution of different photon energies and thus, the approximate values of $\lambda$ and $\sigma_{\mathrm{KN}}$ for the $120-\mathrm{kV}$ polychromatic beam in Eqn. (5) cause blurring in the retrieved image. It has been shown that employing this retrieval method with a monochromatic x-ray beam at the synchrotron facility, the spatial resolution of the retrieved images does not degrade at all [31]. Furthermore, we predict that by employing a high-resolution photon counting detector in the current imaging setup, the contributions of various photon energies in a polychromatic beam can be limited and the PAD-based method would further provide accurate phase retrieval maps without degrading the spatial resolution. 
It is worth to mention that the phase sensitive imaging technique has been adapted to visualize materials with weak attenuation coefficients. The traditional spatial resolution measurements employ a highly attenuating sharp edge or wire composed of a metal. For a metal, the phase effects are not visualized under the diagnostic energy range. These phase effects are the edge enhancements (dark-bright fringes) that occur at the boundaries between components of varying phase shift coefficients. Furthermore, if a metal like tungsten $(W)$ was to be utilized under $60 \mathrm{keV}$, the ratio of $\delta / \beta=74$, while for the PMMA phantom that was used in this study, the ratio of $\delta / \beta=1953$ [46]. Hence, the use of a metal was not feasible in this study. PMMA being a weakly attenuating material $(\mathrm{Z}<10)$ fulfilled one of the conditions of PAD method and, the corresponding phase effects were visualized. The interference patterns occurring at the boundaries resulted in the amplification of signals in the mid-frequencies which has a direct impact on contrast for details corresponding to those frequencies. This trend is also predominantly observed in the LSFs and MTF curves for the CT imaging of lung where a sharp kernel is employed that amplifies the high frequency contents for the better visualization of details like lung nodules [47-49].

The CT reconstruction performed directly with the phase sensitive projections offers an advantage in analyzing the structural inner and outer contours of weakly attenuating tissues. However, these tomograms would not be the maps of the linear attenuation coefficients (LACs) of the tissues. Instead, they may present unusually large or even negative LAC values at interfaces between different tissues [50]. Phase retrieval decodes the phase map from a given phase sensitive projection, and then the 3D images are reconstructed to enhance image contrast and enable to extract quantitative information such as tissue electron density and the so-called phase number [51]. Hence, any phase retrieval method must not degrade the spatial resolution to a great extent so that one could get refined phase maps and tissue's electron density $\left(p_{e}\right)$. In our investigation, the sampling efficiency for the phase retrieved image was about $42 \%(10.6 \mathrm{lp} / \mathrm{mm} / 25 \mathrm{lp} / \mathrm{mm})$ as compared to the $62 \%$ efficiency for the phase sensitive image, a $32 \%$ reduction in sampling efficiency. Other single-distance phase retrieval methods have reported a reduction of more than $60 \%$ in their retrieved images as compared to phase sensitive images [52]. Thus, this retrieval method offers the advantage of offering the quantitative information while working with the polychromatic x-ray beams without greatly degrading the spatial resolution.

With a tube focus spot of $16 \mu \mathrm{m}$, the induced edge gradient blurring is $24 \mu \mathrm{m}$ according to the relation $f=F(M-1)$ [53]. If a large focal spot of $50 \mu \mathrm{m}$ were to be used, the induced edge gradient blurring would increase to $75 \mu \mathrm{m}$ which would increase the FWHM of the LSF curves and degrading the spatial resolution. In our previous work with the $50 \mu \mathrm{m}$ focal spot size, the cutoff resolution for the phase sensitive image was reported to be $12.5 \mathrm{lp} / \mathrm{mm}$ [33]. For translating the phase sensitive technology, operation with the large focal spot size is desired to limit the exposure time associated with microfocus tube's outpower. For efficient mammography or breast imaging system, a cutoff resolution of $8 \mathrm{lp} / \mathrm{mm}$ is enough for accurate diagnosis. For example, the current general electric (GE) digital mammography units use $100 \mu \mathrm{m}$ detector pitch which corresponds to a Nyquist frequency of $5 \mathrm{lp} / \mathrm{mm}$ for the contact modes. In their magnification mode, $M=1.8$, the Nyquist frequency increase to $1.8 \times 5 \mathrm{lp} / \mathrm{mm}=9 \mathrm{lp} / \mathrm{mm}$. The tube focal spot sizes for the breast imaging systems are usually in hundreds of microns, thus their cutoff resolution is always smaller than the Nyquist frequency as dictated by the magnification's blurring. The detector employed in this study has a negative impact on the spatial resolution due to the light spreading that is associated with its scintillator layer. In collaboration with our industrial partners, a clinical phase sensitive imaging system has been developed that utilize a direct detection flat panel system which would further improve the spatial resolution and thus increasing the sampling efficiency of the system. Future studies will be conducted to further optimize this retrieval method to balance the tradeoffs in phase numbers, spatial resolution and image noise. 


\section{Acknowledgements}

The research reported in the publication was supported by the National Cancer Institute of the National Institutes of Health under Award Number R01CA193378. The content is solely the responsibility of the authors and does not necessarily represent the official views of the National Institute of Health. We would like to acknowledge the support of Charles and Jean Smith Chair endowment fund as well.

\section{References}

[1] E. Castelli, M. Tonutti, F. Arfelli, R. Longo, E. Quaia, L. Rigon, D. Sanabor et al., Mammography with synchrotron radiation: First clinical experience with phase-detection technique, Radiology 259(3) (2011), 684-694. doi:10.1148/radiol. 11100745.

[2] M.B. Szafraniec, A.C. Konstantinidis, G. Tromba, D. Dreossi, S. Vecchio, L. Rigon, N. Sodini et al., Synchrotron based planar imaging and digital tomosynthesis of breast and biopsy phantoms using a CMOS active pixel sensor, Physica Medica 31(2) (2015), 192-198. doi:10.1016/j.ejmp.2014.11.003.

[3] K. Bliznakova, P. Russo, Z. Kamarianakis, G. Mettivier, H. Requardt, A. Bravin and I. Buliev, In-line phase-contrast breast tomosynthesis: A phantom feasibility study at a synchrotron radiation facility, Physics in Medicine \& Biology 61(16) (2016), 6243. doi:10.1088/0031-9155/61/16/6243.

[4] P. Baran, S. Pacile, Y.I. Nesterets, S.C. Mayo, C. Dullin, D. Dreossi, F. Arfelli et al., Optimization of propagation-based x-ray phase-contrast tomography for breast cancer imaging, Physics in Medicine \& Biology 62(6) (2017), 2315. doi:10. 1088/1361-6560/aa5d3d.

[5] S.W. Wilkins, Y.I. Nesterets, T.E. Gureyev, S.C. Mayo, A. Pogany and A.W. Stevenson, On the evolution and relative merits of hard x-ray phase-contrast imaging methods, Phil. Trans. R. Soc. A 372(2010) (2014), 20130021. doi:10.1098/ rsta.2013.0021.

[6] S.W. Wilkins, Y.I. Nesterets, T.E. Gureyev, S.C. Mayo, A. Pogany and A.W. Stevenson, On the evolution and relative merits of hard x-ray phase-contrast imaging methods, Phil. Trans. R. Soc. A 372(2010) (2014), 20130021. doi:10.1098/ rsta.2013.0021.

[7] R. Longo, M. Tonutti, L. Rigon, F. Arfelli, D. Dreossi, E. Quai, F. Zanconati, E. Castelli, G. Tromba and M.A. Cova, Clinical study in phase-contrast mammography: Image-quality analysis, Phil. Trans. R. Soc. A 372(2010) (2014), 20130025. doi:10.1098/rsta.2013.0025.

[8] A. Horng, E. Brun, A. Mittone, S. Gasilov, L. Weber, T. Geith, S. Adam-Neumair et al., Cartilage and soft tissue imaging using X-rays: Propagation-based phase-contrast computed tomography of the human knee in comparison with clinical imaging techniques and histology, Investigative Radiology 49(9) (2014), 627-634. doi:10.1097/RLI.0000000000000063.

[9] A. Bravin, P. Coan and P. Suortti, X-ray phase-contrast imaging: From pre-clinical applications towards clinics, Physics in Medicine \& Biology 58(1) (2012), R1.

[10] A. Snigirev, I. Snigireva, V. Kohn, S. Kuznetsov and I. Schelokov, On the possibilities of x-ray phase contrast microimaging by coherent high-energy synchrotron radiation, Review of Scientific Instruments 66(12) (1995), 5486-5492. doi:10. $1063 / 1.1146073$.

[11] S.W. Wilkins, T. Ei Gureyev, D. Gao, A. Pogany and A.W. Stevenson, Phase-contrast imaging using polychromatic hard X-rays, Nature 384(6607) (1996), 335. doi:10.1038/384335a0.

[12] X. Wu and $\mathrm{H}$. Liu, A general theoretical formalism for X-ray phase contrast imaging, Journal of X-Ray Science and Technology 11(1) (2003), 33-42.

[13] X. Wu and H. Liu, Clinical implementation of x-ray phase-contrast imaging: Theoretical foundations and design considerations, Medical Physics 30(8) (2003), 2169-2179. doi:10.1118/1.1593836.

[14] X. Wu and H. Liu, A new theory of phase-contrast x-ray imaging based on Wigner distributions, Medical Physics 31(9) (2004), 2378-2384. doi:10.1118/1.1776672.

[15] P. Tafforeau, R. Boistel, E. Boller, A. Bravin, M. Brunet, Y. Chaimanee, P. Cloetens et al., Applications of X-ray synchrotron microtomography for non-destructive 3D studies of paleontological specimens, Applied Physics A 83(2) (2006), 195-202. doi:10.1007/s00339-006-3507-2.

[16] X. Wu and A. Yan, Phase retrieval from one single phase contrast x-ray image, Optics Express 17(13) (2009), 1118711196. doi:10.1364/OE.17.011187.

[17] L.J. Allen and M.P. Oxley, Phase retrieval from series of images obtained by defocus variation, Optics Communications 199(1-4) (2001), 65-75. doi:10.1016/S0030-4018(01)01556-5.

[18] K.A. Nugent, X-ray noninterferometric phase imaging: A unified picture, JOSA A 24(2) (2007), 536-547. doi:10.1364/ JOSAA.24.000536. 
[19] A. Burvall, U. Lundström, P.A.C. Takman, D.H. Larsson and H.M. Hertz, Phase retrieval in X-ray phase-contrast imaging suitable for tomography, Optics Express 19(11) (2011), 10359-10376. doi:10.1364/OE.19.010359.

[20] A. Yan, X. Wu and H. Liu, Robustness of phase retrieval methods in X-ray phase contrast imaging: A comparison, Medical Physics 38(9) (2011), 5073-5080. doi:10.1118/1.3618731.

[21] T. Weitkamp, D. Haas, D. Wegrzynek and A. Rack, ANKAphase: Software for single-distance phase retrieval from inline X-ray phase-contrast radiographs, Journal of Synchrotron Radiation 18(4) (2011), 617-629. doi:10.1107/ S0909049511002895.

[22] R.C. Chen, H.L. Xie, L. Rigon, R. Longo, E. Castelli and T.Q. Xiao, Phase retrieval in quantitative x-ray microtomography with a single sample-to-detector distance, Optics Letters 36(9) (2011), 1719-1721. doi:10.1364/OL.36.001719.

[23] D. Paganin, S.C. Mayo, T.E. Gureyev, P.R. Miller and S.W. Wilkins, Simultaneous phase and amplitude extraction from a single defocused image of a homogeneous object, Journal of Microscopy 206(1) (2002), 33-40. doi:10.1046/j.13652818.2002.01010.x.

[24] R.C. Chen, L. Rigon and R. Longo, Comparison of single distance phase retrieval algorithms by considering different object composition and the effect of statistical and structural noise, Optics Express 21(6) (2013), 7384-7399. doi:10. 1364/OE.21.007384.

[25] X. Wu, H. Liu and A. Yan, X-ray phase-attenuation duality and phase retrieval, Optics Letters 30(4) (2005), $379-381$. doi:10.1364/OL.30.000379.

[26] X. Wu and H. Liu, X-ray cone-beam phase tomography formulas based on phase-attenuation duality, Optics Express 13(16) (2005), 6000-6014. doi:10.1364/OPEX.13.006000.

[27] H. Liu, Y. Ren, H. Guo, Y. Xue, H. Xie, T. Xiao and X. Wu, Phase retrieval for hard X-ray computed tomography of samples with hybrid compositions, Chinese Optics Letters 10(12) (2012), 121101-121101. doi:10.3788/COL201210. 121101.

[28] X. Guo, X. Liu, M. Gu, C. Ni, S. Huang and B. Liu, Polychromatic X-ray in-line phase-contrast tomography for soft tissue, EPL (Europhysics Letters) 98(1) (2012), 14001. doi:10.1209/0295-5075/98/14001.

[29] Wong, M. Donovan, A. Yan, M. Ghani, Y. Li, L. Fajardo, X. Wu and H. Liu, Dose and detectability improvements with high energy phase sensitive x-ray imaging in comparison to low energy conventional imaging, Physics in Medicine \& Biology 59(9) (2014), N37. doi:10.1088/0031-9155/59/9/N37.

[30] M.U. Ghani, M.D. Wong, D. Wu, B. Zheng, L.L. Fajardo, A. Yan, J. Fuh, X. Wu and H. Liu, Detectability comparison between a high energy $\mathrm{x}$-ray phase sensitive and mammography systems in imaging phantoms with varying glandularadipose ratios, Physics in Medicine \& Biology 62(9) (2017), 3523. doi:10.1088/1361-6560/aa644b.

[31] H. Liu, X. Wu and T. Xiao, Synchrotron-based high-energy X-ray phase sensitive microtomography for biomedical research, Medical Physics 42(10) (2015), 5595-5603. doi:10.1118/1.4929551.

[32] H. Liu, C. Zhang, X. Fan, Y. Duan, T. Xiao, G. Du, Y. Fu, H. Liu and H. Wen, Robust phase-retrieval-based X-ray tomography for morphological assessment of early hepatic echinococcosis infection in rats, PloS ONE 12(9) (2017), e0183396. doi:10.1371/journal.pone.0183396.

[33] D. Wu, A. Yan, Y. Li, M.D. Wong, B. Zheng, X. Wu and H. Liu, Characterization of a high-energy in-line phase contrast tomosynthesis prototype, Medical Physics 42(5) (2015), 2404-2420. doi:10.1118/1.4917227.

[34] P.-C. Lee, Phase retrieval method for in-line phase contrast x-ray imaging and denoising by regularization, Optics Express 23(8) (2015), 10668-10679. doi:10.1364/OE.23.010668.

[35] H. Liu, T. Xiao, H. Xie, Y. Fu, X. Zhang and X. Fan, Nondestructive material characterization of meteorites with synchrotron-based high energy x-ray phase micro-computed tomography, Journal of Physics D: Applied Physics 50(5) (2017), 055301. doi:10.1088/1361-6463/aa4f31.

[36] M.U. Ghani, A. Yan, M.D. Wong, Y. Li, L. Ren, X. Wu and H. Liu, Low dose high energy x-ray in-line phase sensitive imaging prototype: Investigation of optimal geometric conditions and design parameters, Journal of X-Ray Science and Technology 23(6) (2015), 667-682. doi:10.3233/XST-150519.

[37] E. Samei, M.J. Flynn and D.A. Reimann, A method for measuring the presampled MTF of digital radiographic systems using an edge test device, Medical Physics 25(1) (1998), 102-113. doi:10.1118/1.598165.

[38] E. Buhr, S. Günther-Kohfahl and U. Neitzel, Accuracy of a simple method for deriving the presampled modulation transfer function of a digital radiographic system from an edge image, Medical Physics 30(9) (2003), 2323-2331. doi:10.1118/1. 1598673.

[39] E. Samei and M.J. Flynn, An experimental comparison of detector performance for direct and indirect digital radiography systems, Medical Physics 30(4) (2003), 608-622. doi:10.1118/1.1561285.

[40] Z. Zhou, F. Gao, H. Zhao, L. Zhang, L. Ren, Z. Li, M.U. Ghani and H. Liu, Monotone spline regression for accurate MTF measurement at low frequencies, Optics Express 22(19) (2014), 22446-22455. doi:10.1364/OE.22.022446.

[41] L. Ren, Z. Zhou, M.U. Ghani, Y. Li and H. Liu, Method for determining the modulation transfer function of X-ray fluorescence mapping system, Optics Express 22(18) (2014), 21199-21213. doi:10.1364/OE.22.021199. 
[42] M. Stampanoni, Z. Wang, T. Thüring, C. David, E. Roessl, M. Trippel, R.A. Kubik-Huch, G. Singer, M.K. Hohl and N. Hauser, The first analysis and clinical evaluation of native breast tissue using differential phase-contrast mammography, Investigative Radiology 46(12) (2011), 801-806. doi:10.1097/RLI.0b013e31822a585f.

[43] Z. Wang, N. Hauser, G. Singer, M. Trippel, R.A. Kubik-Huch, C.W. Schneider and M. Stampanoni, Non-invasive classification of microcalcifications with phase-contrast X-ray mammography, Nature Communications 5 (2014), 3797. doi:10. 1038/ncomms4797.

[44] A. Momose, W. Yashiro, K. Kido, J. Kiyohara, C. Makifuchi, T. Ito, S. Nagatsuka et al., X-ray phase imaging: From synchrotron to hospital, Phil. Trans. R. Soc. A 372(2010) (2014), 20130023. doi:10.1098/rsta.2013.0023.

[45] M.U. Ghani, M.D. Wong, F.H. Omoumi, B. Zheng, L.L. Fajardo, A. Yan, X. Wu and H. Liu, Detectability comparison of simulated tumors in digital breast tomosynthesis using high-energy X-ray inline phase sensitive and commercial imaging systems, Physica Medica 47 (2018), 34-41. doi:10.1016/j.ejmp.2018.02.012.

[46] CSIRO - website, https://ts-imaging.net/Services/Simple/ICUtilXdata.aspx (accessed 10.26.2017).

[47] J.M. Boone, Determination of the presampled MTF in computed tomography, Medical Physics 28(3) (2001), 356-360. doi: $10.1118 / 1.1350438$

[48] M. Ohkubo, S. Wada, A. Kayugawa, T. Matsumoto and K. Murao, Image filtering as an alternative to the application of a different reconstruction kernel in CT imaging: Feasibility study in lung cancer screening, Medical Physics 38(7) (2011), 3915-3923. doi:10.1118/1.3590363.

[49] J.G. Ott, F. Becce, P. Monnin, S. Schmidt, F.O. Bochud and F.R. Verdun, Update on the non-prewhitening model observer in computed tomography for the assessment of the adaptive statistical and model-based iterative reconstruction algorithms, Physics in Medicine \& Biology 59(15) (2014), 4047. doi:10.1088/0031-9155/59/4/4047.

[50] X. Wu and H. Liu, X-ray cone-beam phase tomography formulas based on phase-attenuation duality, Optics Express 13(16) (2005), 6000-6014. doi:10.1364/OPEX.13.006000.

[51] X. Wu, H. Liu and A. Yan, Phase-contrast X-ray tomography: Contrast mechanism and roles of phase retrieval, European Journal of Radiology 68(3) (2008), S8-S12.

[52] A. Sarno, G. Mettivier, B. Golosio, P. Oliva, G. Spandre, F. Di Lillo, C. Fedon, R. Longo and P. Russo, Imaging performance of phase-contrast breast computed tomography with synchrotron radiation and a CdTe photon-counting detector, Physica Medica 32(5) (2016), 681-690. doi:10.1016/j.ejmp.2016.04.011.

[53] J.T. Bushberg and J.M. Boone, The Essential Physics of Medical Imaging. Chapter-7, Lippincott Williams \& Wilkins, 2011, pp. 208-209. 